\title{
Extracting Power from Sub-Wavelength Apertures by Using Electrically Small Resonators: Phenomenology, Modeling, and Applications
}

\author{
Filiberto Bilotti, Luca Di Palma, Davide Ramaccia, \\ Alessandro Toscano, and Lucio Vegni \\ Department of Applied Electronics \\ "Roma Tre" University \\ Via della Vasca Navale, 84 - 00146 - Rome - Italy \\ bilotti@uniroma3.it
}

\author{
Damla Ates and Ekmel Ozbay \\ Nanotechnology Research Centre \\ Bilkent University \\ Ankara, Turkey
}

\begin{abstract}
In this contribution, we review our recent work on the extraction of the electromagnetic power from electrically small apertures by using metamaterial-inspired resonators. First, we present an antenna interpretation of the power transmission through sub-wavelength apertures and discuss the questioned concept of "enhanced transmission". Then, we present the socalled "connected bi-omega particle" and the related analytical model. After that, exploiting proper numerical and experimental examples, we also show that the electromagnetic response of such a particle is not influenced by the surrounding environment. This unique property makes the particle a suitable candidate for the implementation of microwave components based on the selective power extraction from electrically small apertures. Finally, the application of the proposed concepts to the design of innovative microwave components, such as waveguide filters, diplexers, power-splitters, modal filters, horn antennas, etc. will be considered and demonstrated through proper numerical and experimental results.
\end{abstract}

Connected bi-omega particle; metamaterial-inspired resonators; power transmission through sub-wavelength aperture; enhanced transmission

\section{INTRODUCTION}

The employment of metamaterials in microwave components has led to several interesting design possibilities and opened the door to conceptually new devices and applications [1]. In the field of radio-frequency and microwave antennas, one of the most tangible advantage enabled by metamaterials certainly refers to the miniaturization of the radiator dimensions. Typical applications concern dipole and microstrip antennas, whose electrical dimensions can be squeezed by loading electrically short radiators with a single or a few metamaterial-inspired resonators [2]-[4]. In these cases, what happens is that a short antenna, which is a highly inefficient and mismatched radiator by itself, is brought to a resonance condition by the presence of the electrically small resonator(s) and is matched to its feeding line through the reactive impedance matching enabled by the same resonator(s).
This approach, which has been successfully demonstrated for linear and microstrip antennas, can be extended also to the case of electrically small aperture antennas. Typically, in order to efficiently extract power from an electrically small aperture, which is an highly inefficient radiator, one can place a conventional resonating element across the aperture. However, this solution results in a quite bulky setup, which is in conflict with the final goal of having a miniaturized efficient aperture. In other words, in order to make a sub-wavelength aperture radiating efficiently, the additional resonator should have a resonant length (typically half wavelength). As it was the case in the miniaturization of linear antennas, metamaterial concepts can play a significant role also in this application field.

Miniaturized resonators as the ones used for metamaterial inclusions (e.g. split-ring resonators, etc.), in fact, can be successfully used for this purpose. For instance, it has been demonstrated that one or two split-ring resonators placed across an electrically small aperture allow extracting power efficiently from the aperture, without altering the compactness of the entire setup [5]-[8].

In this contribution, we review our recent results obtained employing the so-called connected bi-omega particle to efficiently extract power from a sub-wavelength aperture. We show that the resonant frequency of such a particle is slightly influenced by the surrounding environment and can be predicted by using a simple analytical model. In this way, the synthesis of the particle becomes a straightforward task, allowing the design of a new family of microwave components, including electrically small aperture antennas, self-filtering horn radiators, waveguide microwave diplexers, filters, (un)balanced and switchable power splitters, etc.

\section{THE CONNECTED BI-OMEgA PARTICLE}

The connected bi-omega particle we consider in this paper is sketched in Fig. 1a. It consists of two reversed metallic omega pairs, printed on the two sides of a dielectric board. From geometrical considerations, it results that this 
symmetrical structure supports two fundamental modes, characterized by odd and even electric field distributions, respectively. The odd mode is quite interesting because it is characterized by a strong excitation of the two strips connecting the omegas at each board side.

Due to the balanced configuration of the particle and to the localization of the field within the particle shape, the resonant frequency of the modes are expected to be independent from the surrounding environment and depend only on the geometrical parameters of the particle and the geometrical/electrical parameters of the board.

Since the particle size is a small fraction of the wavelength at the resonant frequency, it is possible to model its electromagnetic behavior through a quasi-static model that can be easily obtained by exploiting the earlier results on individual helical and omega particles and on the helical particle pair. The full details about the analytical model will be presented at the conference. Here we would like to remark only that the agreement between the analytical model and the full-wave simulations is excellent and that the expected independence of the resonant frequencies of the particle with respect to the surrounding environment is verified, as shown in Fig. 1b. As an example, in the figure we report the cases of the particle in free-space and placed across an electrically small slit drilled in a metallic plate. The related experimental results have been recently published in [9].

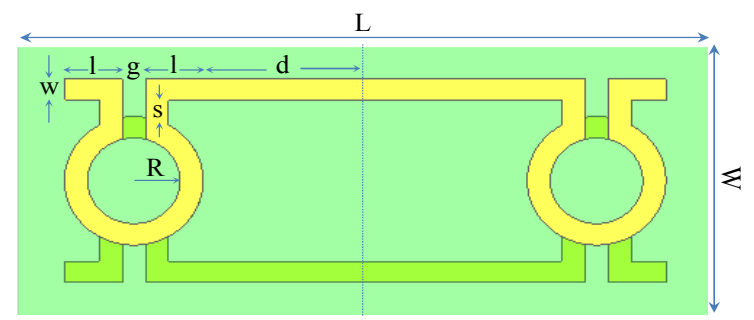

a)

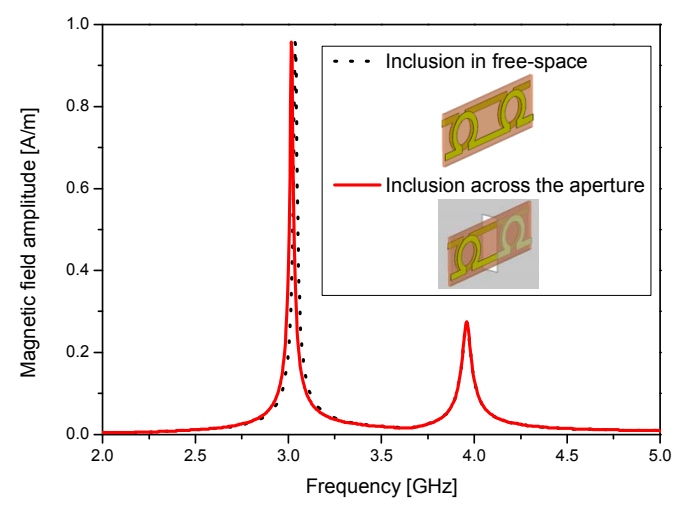

b)

Figure 1. a) Geometrical sketch of the connected bi-omega particle. b) Amplitude of the magnetic field recorded by the probes at the centres of the loops in the cases of a slab in free-space and across a slit in a metallic screen.

\section{APPLICATIONS}

As previously anticipated, the proposed structure can be successfully used to conceive a new family of microwave components characterized by compact dimensions and selective transmission properties. A typical application scenario can be in the implementation of microwave components for satellite communications. They are commonly realized in waveguide technology and, thus, operate over a broad frequency band. However, while for other applications broadband operation is desirable, this is not the case in satellite systems, where narrowband signals are used and noise must be filtered out efficiently. In such systems, noise reduction is typically accomplished by using bulky and expensive additional filters, that can be easily replaced by the structure reported in the previous section. The proposed solution, in fact, is cheaper, lighter, and does not require additional space.

The connected bi-omega particle can be easily integrated inside any component. In Fig. 2, we show a new self-filtering horn antenna for satellite applications we have tested and fabricated [10]. The radiator is based on a regular WR-62 horn, having a metallic screen inserted at the horn throat, between the feeding waveguide and the flared aperture. We have drilled a vertical rectangular slit on the screen (the long side of the slit is in the E-plane) and placed a dielectric slab across the slit itself.

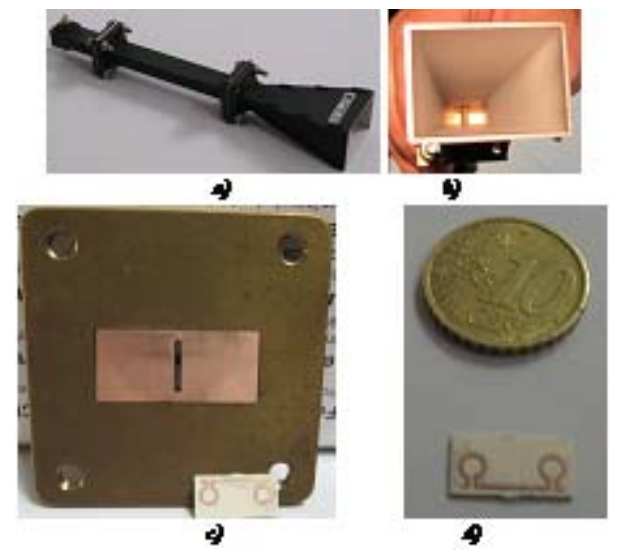

a)

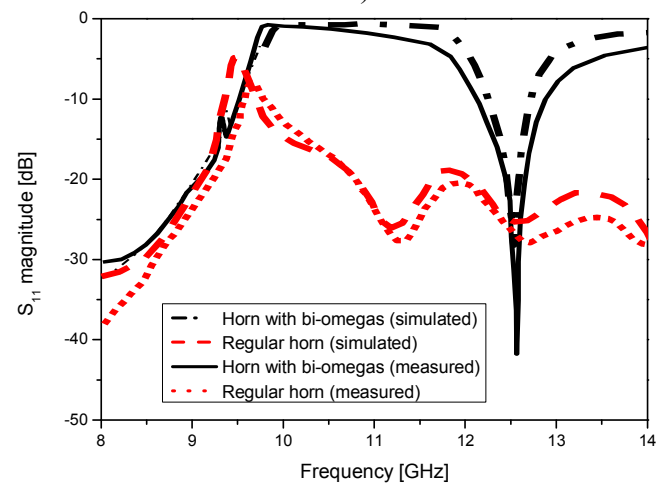

b)

Figure 2. a) Self-filtering horn antenna. b) Reflection coefficient of the horn with and without the connected bi-omega particle placed at its throat. 
On the two sides of the slab, we have printed the metallic bi-omega particle with appropriate dimensions in order to resonate in a sub-band of the operating frequency range of the WR-62 horn. At the particle resonance, signals are allowed to pass through the screen, reducing, thus, the operating frequency band of the WR-62 horn.

In Fig. 3, we show the design of a waveguide diplexer [11]. The component consists in a junction between three waveguides with the same cross-section. At either of the junction sections between the feeding waveguide and the two output ones, we place a set of connected bi-omega particles. The two sets of particles have different dimensions, in order to resonate at two different frequencies, enabling, thus, the frequency selective transmission required for the diplexer operation.

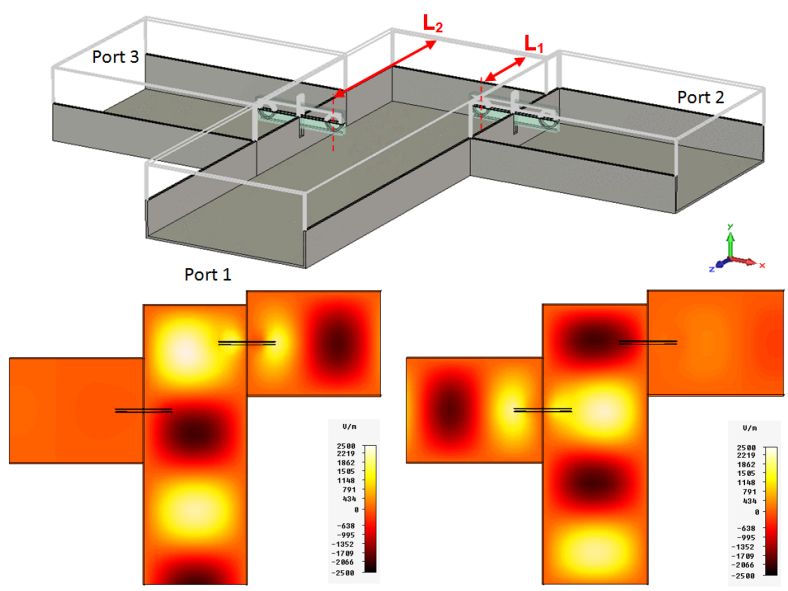

a)

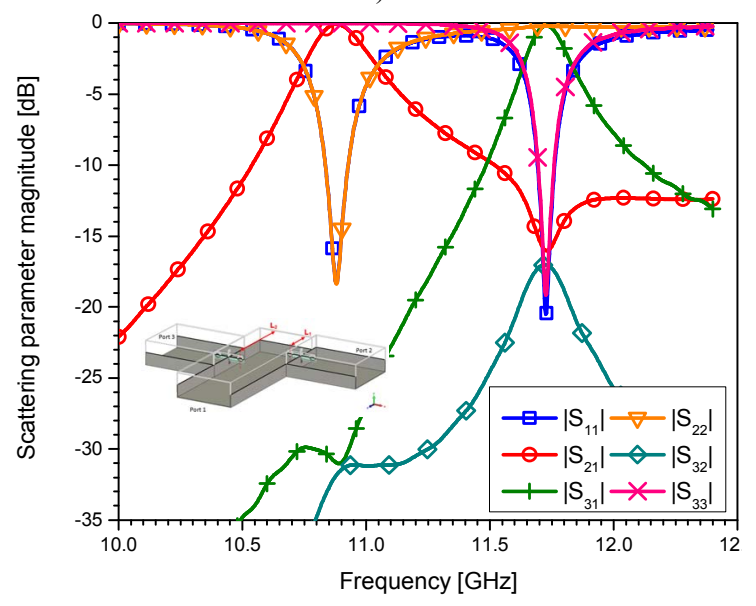

b)

Figure 3. a) Diplexer sketch and field distribution at the two different resonant frequencies of the connected bi-omegas at Ports 2 and 3, respectively. b) Magnitude of the $\mathrm{S}$ parameters of the diplexer.

Exploiting the same concepts, balanced, unbalanced and tunable power-splitters [12]-[13] have also been demonstrated. In this case, the component consists again in a junction between three waveguides with the same cross-section. This time, however, the two sets of particles are identical. While the balanced components are obtained by exploiting the symmetry of the structure and aligning the two sets of particles along the same axis, the unbalanced ones are obtained by either breaking the symmetry (e.g. introducing a relative shift between the two sets of particles) or introducing proper switches in the design. As an example, in Fig. 4 we show an unbalanced tunable power splitter obtained by placing the switches at proper locations on the omega particles.

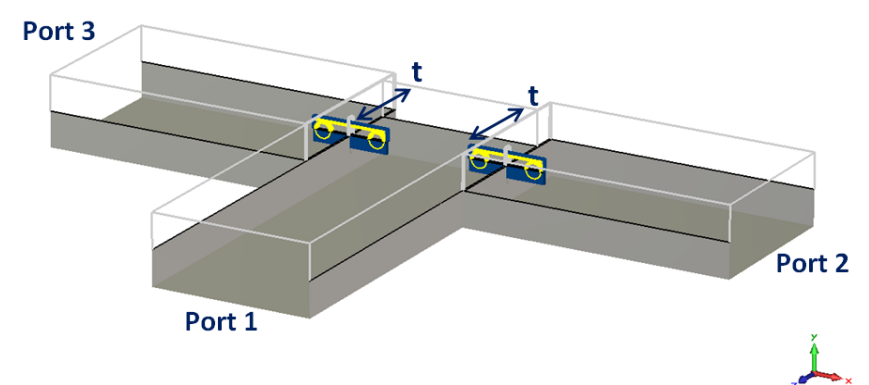

a)

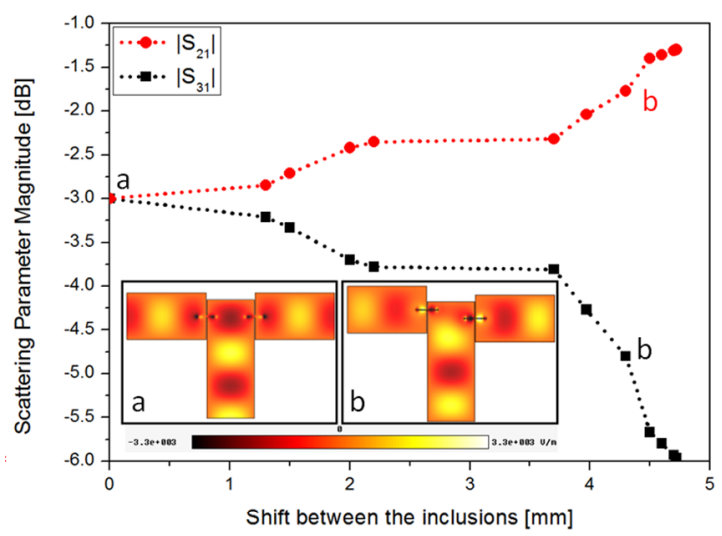

b)

Figure 4. a) a) Power splitter sketch. b) Magnitude of the simulated $\mathrm{S}_{21}$ and $\mathrm{S}_{31}$ parameters of the unbalanced power splitter varying the shift $\mathrm{d}$ between the two bi-omega particles. In the insets a) and b) we show the maps of the ycomponent of the electric field for the balanced $(d=0 \mathrm{~mm})$ and one unbalanced $(\mathrm{d}=4.3 \mathrm{~mm})$ case.

More details on the design procedures and operation of the components of Figs. 2-4 and further applications will be presented at the congress.

\section{CONCLUSIONS}

In this contribution, we have reviewed our recent work on the power extraction from electrically small apertures by using metamaterial-inspired resonators. Particularly, we have reviewed the operation principles and the main properties of the connected bi-omega particle. We have also shown that the electromagnetic response of such a particle is not influenced by the surrounding environment and that this unique property can be successfully used for the design of microwave components 
2012 Loughborough Antennas \& Propagation Conference

based on the selective power extraction from electrically small apertures. Finally, the application of the proposed concepts to the design of innovative microwave components, such as waveguide filters, diplexers, power-splitters, modal filters, horn antennas, etc. has been considered and demonstrated through proper numerical and experimental results.

\section{REFERENCES}

[1] F. Bilotti, and L. Sevgi, "Metamaterials: definitions, properties, applications, and FDTD-based modeling and simulation," Int. J. RF Microw. CAE, Vol. 22, pp. 422-438, 2012.

[2] A. Erentok and R. Ziolkowski, "Metamaterial-Inspired Efficient Electrically Small Antennas,” IEEE Trans. Antennas Propagat., vol. 56, pp. 691-706, 2008.

[3] R.W. Ziolkowski and A. Erentok, "Metamaterial-based efficient electrically small antennas," IEEE Trans. Antennas Propagat., vol. 54, pp. 2113-2130, 2006.

[4] F. Bilotti, A. Alù, and L. Vegni, "Design of Miniaturized Metamaterial Patch Antennas with $\mu$-Negative Loading," IEEE Trans. Antennas Propagat., vol. 56, pp. 1640-1647, 2008.

[5] F. Bilotti, L. Scorrano, E. Ozbay, and L. Vegni, "Enhanced transmission through a sub-wavelength aperture: Resonant approaches employing metamaterials," J. Opt. A, Vol.11, 114029, 2009.

[6] K. Aydin, A.O. Cakmak, L. Sahin, Z. Li, F. Bilotti, L. Vegni, and E. Ozbay, "Split-ring-resonator-coupled enhanced transmission through a single subwavelength aperture," Phys. Rev. Lett., Vol. 102, pp. 013904, 2009.

\section{2 - 13 November 2012, Loughborough, UK}

[7] K.B. Alici, F. Bilotti, L. Vegni, and E. Ozbay, "Optimization and tunability of deep subwavelength resonators for metamaterial applications: complete enhanced transmission through a subwavelength aperture," Opt. Expr., Vol. 17, pp. 5933-5943, 2009.

[8] A.O. Cakmak, K. Aydin, E. Colak, Z. Li, F. Bilotti, L. Vegni, and E. Ozbay, "Enhanced transmission through a sub-wavelength aperture using metamaterials," Appl. Phys. Lett., Vol. 95, 052103, 2009.

[9] D. Ates, F. Bilotti, A. Toscano, and E. Ozbay, "Experimental demonstration of the enhanced transmission through circular and rectangular sub-wavelength apertures using omega-like split-ring resonators," Photonics and Nanostructures - Fundamentals and Applications (to appear)

[10] F. Bilotti, L. Di Palma, D. Ramaccia, and A. Toscano, "Self-filtering low-noise horn antenna for satellite applications," IEEE Antennas Wireless Propagat. Lett., Vol. 11, pp. 354-357, 2012.

[11] L. Di Palma, F. Bilotti, A. Toscano, and L. Vegni, "Design of a waveguide diplexer based on connected bi-omega particles," IEEE Microw. Wireless Compon. Lett., Vol. 22, No. 3, pp. 126-128, 2012.

[12] L. Di Palma, F. Bilotti, A. Toscano, and L. Vegni, "Design of a waveguide power splitter based on the employment of bi-omega resonators," Microw. Opt. Technol. Lett., Vol. 54, No. 9, pp. 20912095, 2012.

[13] L. Di Palma, D. Ramaccia, F. Bilotti, A. Toscano, S. Scafè, and G. Guarnieri, "Balanced, Unbalanced, and Tunable Waveguide Power Splitters Based on Connected Bi-Omega Particles," submitted to IEEE Microw. Wireless Compon. Lett., 2012 